\title{
Summary of: Models of practice organisation using dental therapists: English case studies
}

\section{FULL PAPER DETAILS}

1PhD Student, Institute of Psychology, Health and Society, University of Liverpool; ${ }^{2 *}$ Senior Clinical Lecturer/Honorary Consultant, Department of Health Services Research, School of Dentistry, University of Liverpool

${ }^{*}$ Correspondence to: Dr Rebecca Harris, Waterhouse Building, Block B, 1st Floor, Room B113, 1-5 Brownlow Street, Liverpool L69 3GL

Tel: +44 (0) 151795 5334; Fax: +44 (0)151 7945604 Email:harrisrv@liv.ac.uk

Online article number E6

Refereed Paper - accepted 4 March 2011

DOI: 10.1038/sj.bdj.2011.624

${ }^{\circ}$ British Dental Journal 2011; 211: E6

\author{
VERIFIABLE CPD PAPER
}

Introduction A new dental remuneration system based on bands of activity has changed the reward system operating in dental practices and influenced practitioner behaviour in relation to the delegation of tasks to English dental therapists (DTs). Since dental practitioners operate as independent contractors they are free to innovate. A variety of models incorporating DTs in general practice teams exist, some of which may overcome the apparent delegation constraints embedded within this system of remuneration. Objectives To describe the way different practices are organised to take account of DTs in their teams and identify whether any of these models address delegation disincentives arising from the system of remuneration. Method A purposive sample of six dental practices was identified, comprising two small, two medium and two large dental practices, including a variety of models of practice organisation. Semi-structured interviews were carried out with principal dentists, associate dentists, DTs, practice managers and dental hygienists (35 participants in total). A thematic analysis was applied to interview transcripts. Results The six dental practices demonstrated six different models of practice organisation which could be grouped into 'practice payment' and 'dentist payment' models according to whether the salary costs of the DT were met by a central practice fund or from the income of individual dentists in the team. In both of the large practices only some of the dentists in the team referred work to the DT because of reimbursement issues. In two practices the system was perceived to be satisfactory to all parties, one of these being a single-handed practice with two DTs. Conclusion Although the remuneration system contained some potential disincentives to DT delegation, some practices innovated in their organisations to overcome these issues.

\section{EDITOR'S SUMMARY}

Paul Batchelor, the Commentary writer for this research paper quotes the adage 'if skill mix is the answer, what is the question?' It is an expression that I have not heard before in this context. However, it brings to mind a piece of wonderfully witty graffiti I saw many years ago when one of the major political parties used the election slogan '[name of party] is the answer'; to which a sharp-penned opponent had appended the riposte 'in which case it must have been a very silly question'.

That is not to say that I think the purpose behind this research is silly or misplaced in the least but the quoted adage does raise very important questions in relation to the reasons for which we are supposedly pursuing skill mix within oral care delivery in the UK. The authors 'drill down' (I believe is the current parlance) into the organisation of practices who are employing dental therapists and add to our accumulating knowledge of how well, or otherwise, they are accepted and economically effective.

But what is the question? Are we heading in this direction because of economic constraints which may be alleviated by the better value brand of dental team members clinically enabled by division of labour? Is it a function of the anticipation of falling disease levels dictating a broader divide between less complex treatment need and very specialised dental treatment?

Or is it that we are driven towards 'using' dental therapists because, quite simply, they are there? We have published a number of papers in the $B D J$ in recent years looking at various aspects of the employment of dental therapists which have thrown considerable light on the subject and which have been read and referenced with great interest in other countries where such team members either do not exist at all or have considerably curtailed fields of operation. To be truthful though, I am not sure that any of them have pondered this fundamental question; which in itself is intriguing.

The full paper can be accessed from the $B D J$ website (www.bdj.co.uk), under 'Research' in the table of contents for Volume 211 issue 3.

Stephen Hancocks Editor-in-Chief

DOI: 10.1038/sj.bdj.2011.649 


\section{TO ACCESS THE BDJ WEBSITE TO READ THE FULL PAPER:}

- BDA Members should go to www.bda.org.

- Click the 'login' button on the right-hand side and enter your BDA login details.

- Once you have logged in click the 'BDJ' tab to transfer to the BDJ website with full access.

IF YOUR LOGIN DETAILS DO NOT WORK:

- Get a password reminder: go to www.bda.org, click the login button on the right-hand side and then click the forgotten password link.

- Use a recommended browser: we recommend Microsoft Internet Explorer or Mozilla Firefox.

- Ensure that the security settings on your browser are set to recommended levels.

IF YOU HAVE NOT YET SIGNED UP TO USE THE BDA WEBSITE:

- Go to www.bda.org/getstarted for information on how to start using the BDA website.
IN BRIEF

- Addresses practice organisation issues which act as barriers to successfully incorporating dental therapists (DTs) into dental practice teams.

- Identifies two main approaches to reimbursing DTs: out of overall practice revenue or by deductions from gross income of individual dentists.

- Models include examples of using DTs in small teams.

\section{COMMENTARY}

One of the key policy decisions within a care system lies in identifying and ensuring that there is an appropriate skilled workforce to achieve the stated goals of a system. Historically the oral healthcare delivery system has relied on dentists to provide oral healthcare. Recent developments suggest this may be changing: there appears to be, at least in the United Kingdom, a decision that a workforce structure of differing grades of dental personnel would be a more apt solution to help meet future oral health needs.

The current paper has begun to explore some of the wider contextual issues that would need to be overcome if such a policy is to work: the adage 'if skill mix is the answer, what is the question?' remains all too true. Using qualitative techniques the authors have identified practices of different organisational structure (with a heavy emphasis on remuneration methods) to try and identify some of the barriers to ensuring that dental therapists can be used in an effective manner. One of the difficulties faced in attempting to answer the question is identified in the wide range of structural issues outlined. These include remuneration arrangements, referral arrangements and employment status, many of which practice principals would be aware of when using associates.

The authors have also challenged the assumption that larger practices ensure that the care delivery arrangement functions efficiently: it is more about good management (and leader- ship) within the structure. This is an issue that would be worthy of greater discussion if dental therapists are to become an integral component of a care delivery system.

What the authors have also recognised is that while reimbursement plays a key role when designing a care delivery system, it is not the only factor. Indeed, as the authors have commented, the now discredited UDA system has had some impact on their effective use but its demise has already been signalled. If a policy of a workforce in which dental therapists, or indeed other dental care professionals are to play a major part in the future is to succeed, the paper has highlighted that the remuneration system is simply one of a number of elements that will need to be thought through.

\section{P. Batchelor}

Consultant in Dental Public Health, Thames Valley PCT Consortium Course Director, Leadership and Management programme, FGDP(UK) Honorary Senior Lecturer, Dental Public Health Unit, University College London

\section{AUTHOR QUESTIONS AND ANSWERS}

1. Why did you undertake this research? We were aware that although dental therapists work successfully in many dental practices in England, there were feelings by some practitioners that having a dental therapist in the team weakened the financial performance of the practice. There were also reports that the recent shift to a UDA remuneration system had exacerbated the issue. We were interested to explore the issues in more depth, and since dental practices are free to innovate we wanted to see if any solutions had been found.

2. What would you like to do next in this area to follow on from this work?

A central issue is whether dental therapists are fully incorporated into the dental team, with their performance evaluated with regard to their contribution to team performance. There appear to be fewer concerns over the financial contribution of dental hygienists than with dental therapists. An in-depth comparison between the two roles would make an interesting study. 